\title{
Estimate of free electron laser gain length in the presence of electron beam collective effects
}

\author{
S. Di Mitri \\ Elettra Sincrotrone Trieste, 34149 Basovizza, Trieste, Italy \\ S. Spampinati \\ Department of Physics, University of Liverpool, Liverpool L69 7ZX, \\ United Kingdom and Cockroft Institute, Sci-Tech Daresbury, \\ Warrington WA4 4AD, United Kingdom \\ (Received 7 May 2014; published 12 November 2014)
}

\begin{abstract}
We analytically estimated the three-dimensional free electron laser (FEL) power gain length's increase due to the collective effects of an ultrarelativistic electron beam, namely, geometric transverse wakefield, coherent synchrotron radiation, and microbunching instability. We showed that the gain length is affected by an increase of the electron beam projected emittance, even though the slice (local) emittance is preserved. We also proved that the minimum gain length and the maximum of output power may notably differ from the ones derived when collective effects are neglected. Finally, we demonstrated that our model may be handy for a parametric study of electron beam six-dimensional brightness and FEL performance as a function, e.g., of the bunch length compression factor, the accelerator alignment tolerances, and the optics design.
\end{abstract}

DOI: $10.1103 /$ PhysRevSTAB.17.110702

PACS numbers: 29.20.Ej, 29.27.Bd, 41.60.Cr

\section{WORK PLAN}

The optimization of a free electron laser (FEL) design usually involves minimization of the FEL power gain length [1]. For many years, FEL theories that include three-dimensional (3D) effects (although they should be more correctly called 2D) have been proposed $[2,3]$ to predict the gain length and other FEL parameters when the 1D approximation falls short of a realistic physics model. In recent times, two analytical models [4,5] have been commonly used to estimate the so-called 3D gain length, $L_{g, 3 \mathrm{D}}$, which takes into account the electron beam finite transverse emittance and energy spread, in self-amplified spontaneous emission (SASE) FELs [6,7]. In these models, the emittance and the energy spread are those of a longitudinal slice of the electron bunch. The slice is assumed to be as long as the FEL cooperation length [6], thus typically much shorter than the total bunch duration. An additional 3D effect, that is, the impact of transverse deviations of the electron bunch's trajectory from its reference path in the undulator on the FEL 1D gain length $L_{g, 1 \mathrm{D}}$, was modeled in Ref. [8] and experimentally studied in Ref. [9].

Starting from the formalism developed in the cited works $[4,5,8]$ and from our prior study [10], in this article: (i) We

Published by the American Physical Society under the terms of the Creative Commons Attribution 3.0 License. Further distribution of this work must maintain attribution to the author(s) and the published article's title, journal citation, and DOI. analytically evaluate the electron beam 6D energynormalized brightness $B_{n, 6 \mathrm{D}}$ in the presence of a short-range geometric transverse wakefield (GTW) in accelerating structures and coherent synchrotron radiation (CSR) emitted in magnetic compressors. We extend our previous study [10] to include the analytical estimate of the final slice energy spread when microbunching instability (MBI) is suppressed with a laser heater [11]. This estimate makes use of the analytical model for the MBI given in Refs. [12,13]. (ii) We show that the physical picture proposed in Ref. [8] for the beam motion in an undulator also applies to angular perturbations caused by GTW and CSR in the accelerator. Consequently, we establish an explicit connection between the FEL performance, so far predicted only on the basis of the electron bunch's slice parameters, and a more complete set of sources of $B_{n, 6 \mathrm{D}}$ degradation that includes projected beam parameters. (iii) An analytical formula is given for estimating the SASE FEL 3D power gain length's increase due to collective effects, the power saturation length, and the peak power at saturation. We extend the discussion beyond SASE to the case of externally seeded FELs.

The article is organized as follows. In Sec. II, a novel definition of the SASE FEL 3D power gain length, hereafter named $L_{g, \text { coll }}$, is given, which accounts for the electron beam transverse projected emittance. The consistency of this definition with the one given by Xie [4] is proven with analytical and numerical results. In Sec. III, the slice energy spread at the undulator entrance is estimated in the case of MBI suppressed with a laser heater. The predicted value of the projected emittance and the slice energy spread are used 
in Sec. IV to evaluate $B_{n, 6 \mathrm{D}}$ for two realistic linac models. In Sec. V, $L_{g \text {,coll }}$ and other FEL-related parameters are scanned vs the bunch length compression factor. Their sensitivity to the accelerator alignment tolerances and the compressor optics design is eventually provided. Conclusions are given in Sec. VI.

\section{COLLECTIVE EFFECTS IN THE TRANSVERSE PLANES}

\section{A. New definition of 3D gain length}

GTW and CSR offset individual "macroslices" (bunch longitudinal portions) in both configuration and velocity spaces. The macroslices are modeled to be as long as several cooperation lengths, since GTW and CSR-induced transverse kicks are typically correlated with $z$, the longitudinal coordinate internal to the bunch, on the length scale of a few to hundreds of microns (see [14] and references therein). The cooperation length of extreme ultraviolet (EUV) and x-ray FELs, which are of interest here, is typically shorter than $1 \mu \mathrm{m}$. Neglecting for the moment any slice emittance growth from the injector to the undulator, the projected emittance growth is entirely due to mismatch of the bunch macroslices in the transverse phase space. We take this growth into account through the mechanism described by Tanaka, Kitamura, and Shintake [8]. In that work, the authors identify two distinct processes that increase $L_{g, 1 \mathrm{D}}$. One is a lack of overlapping between the spontaneous undulator radiation, whose wave front follows the electrons' local direction of motion, and the FEL radiation, whose wave front is preserved when the electrons are transversally kicked by lattice errors. The other process is electrons' bunching smearing due to longitudinal dispersion of electrons transversally kicked by lattice errors. The latter effect is more relevant to the gain length's increase. In the literature, the first effect is also referred to as the (lack of) electrons-photons spatial overlap along the undulator. The second one describes the accumulation of phase error between electrons and radiation by virtue of the slowing down of the electrons due to their local angular divergence.

We recognize that the electrons' angular divergence has two contributions: One is incoherent and due to the finite beam emittance as depicted in Xie's [4] and Saldin's [5] models; the other is coherent, being the tilt of the macroslice centroids with respect to the reference trajectory. The coherent divergence adds to (and, in some cases, surpasses) the incoherent one and may amplify the effect of bunching smearing (see Fig. 3 in Ref. [8]). In order to take into account the coherent motion of electrons, we apply the physical picture depicted in Ref. [8] to individual macroslices. Each macroslice is transversally kicked by collective effects in the linac and thus moves along the undulator on a different trajectory than other macroslices, as shown in Fig. 1.

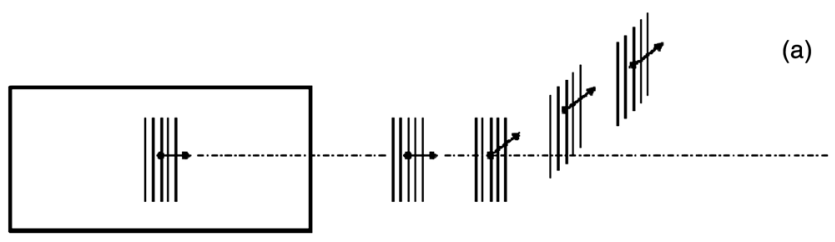

(b)
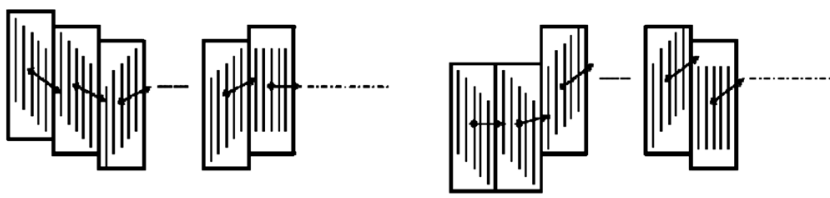

FIG. 1. Effect of a transverse kick on electrons in an undulator. In Tanaka's picture [8] (a), all electrons in the bunch follow the same direction of motion as a whole, before (left side) and after the kick. In our picture (b), instead, different macroslices in the same bunch follow different directions of motion along the undulator by virtue of their initial different launching conditions. These were determined by collective effects in the upstream accelerator. In the sketch, solid lines define the bunch (a) or a macroslice (b); arrows indicate the electrons' direction of motion; vertical bars identify the FEL microbunch wave front orientation.

We call $\sqrt{\left\langle\theta_{\text {coll }}^{2}\right\rangle}$ the rms angular divergence of the macroslice centroids at the undulator. Being a quantity averaged over the bunch duration, $\sqrt{\left\langle\theta_{\text {coll }}^{2}\right\rangle}$ is an indicator of the mismatch of the macroslices in the transverse phase space, projected onto the $z$ coordinate. We assume that the charge transverse distribution at the undulator is matched to some design Twiss parameters (the validity of this assumption is discussed below) and that a smooth optics is implemented all along the undulator line: $\beta_{u}$ is the average betatron function, and its variation is small along the undulator. Thus, the determinant of the so-called "sigma matrix" [15] computed at the undulator provides the beam projected emittance as a function of $\sqrt{\left\langle\theta_{\text {coll }}^{2}\right\rangle}$ and $\beta_{u}$ :

$$
\varepsilon_{n, f} \approx \varepsilon_{n, 0} \sqrt{1+\frac{\gamma \beta_{u}\left\langle\theta_{\mathrm{coll}}^{2}\right\rangle}{\varepsilon_{n, 0}}},
$$

with $\varepsilon_{n, 0}$ and $\varepsilon_{n, f}$ being the initial (unperturbed) normalized and the final normalized emittance in the plane of interest, respectively, and $\gamma$ the relativistic Lorentz factor at the undulator. Finally, we revise Tanaka's formula for the gain length [see [8], Eq. (9)] and make the following ansatz to estimate the 3D gain length in the presence of collective effects:

$$
L_{g, \text { coll }} \approx \frac{L_{g, 3 \mathrm{D}}}{1-\pi\left\langle\theta_{\mathrm{coll}}^{2}\right\rangle / \theta_{\mathrm{th}}^{2}} .
$$

$L_{g, 3 \mathrm{D}}$ is the 3D power gain length in the sense of Xie [4]; $\theta_{\text {th }}=\sqrt{\lambda / L_{g, 3 \mathrm{D}}}$ and $\lambda$ is the FEL wavelength. The electron beam slice transverse emittance and the slice energy spread 
at the undulator are taken into account in $L_{g, 3 \mathrm{D}}$; the information on the degradation of the projected emittance is brought about by $\left\langle\theta_{\text {coll }}^{2}\right\rangle$. The range of application of Eq. (2) is $\left\langle\theta_{\text {coll }}^{2}\right\rangle<\theta_{\text {th }}^{2} / \pi$. Larger values of $\left\langle\theta_{\text {coll }}^{2}\right\rangle$ are assumed to inhibit the FEL process.

It is worth to remark that Eq. (2) relies on two realistic assumptions. First, we suppose that the motion of the misaligned macroslices is governed by Twiss functions and phase advance close to the design ones. This is true either for small GTW- and CSR-induced deformation of the transverse phase space or when, more generally, the particle transverse distribution is rematched to the design Twiss parameters before entering the undulator. Second, we neglect the angular kicks given by either the undulator segments or the quadrupole magnets interleaving the segments. In other words, we assume that the effect of angular kicks by the magnetic lattice on the misaligned macroslices is negligible with respect to the effect of the macroslices' misalignment on the gain length. We also neglect any dependence of the undulator parameter with the electron bunch transverse position.

\section{B. Dependence on beam optics}

Equation (2) aims to generalize Xie's formalism, so that $L_{g, \text { coll }}$ reduces to $L_{g, 3 \mathrm{D}}$ for either null collective effects $\left\langle\theta_{\text {coll }}^{2}\right\rangle=0$ or large $\beta_{u}$, for any preset emittance growth [see Eq. (1)]. The dependence on $\beta_{u}$ is explained as follows. We assume an electron beam whose normalized emittance grows along the linac according to $\Delta \varepsilon_{n}=\varepsilon_{n, f}-\varepsilon_{n, 0}$. If such a growth concerns only the slice emittance, the gain length will be $L_{g, 3 \mathrm{D}}$ according to Ref. [4]. If, instead, the emittance growth is only the projected one, and the slice emittance is preserved at the injector level, the gain length will be $L_{g \text {,coll }}$ in Eq. (2). We will see in the remainder of this article that the projected growth $\Delta \varepsilon_{n}$ is uniquely determined by the initial beam parameters and the linac setting and, as already said, it is due to the bunch slices' misalignment in the transverse phase space. Then, if $\beta_{u}$ is large, as in a weak focusing undulator lattice, the macroslices will tend to overlap in angular divergence, that is, $\left\langle\theta_{\text {coll }}^{2}\right\rangle \rightarrow 0$, as shown in Fig. 2(d). In this case, we expect $L_{g \text {,coll }}$ to approach $L_{g, 3 \mathrm{D}}$. On the contrary, a small $\beta_{u}$ as due, e.g., by strong focusing, will force the macroslice centroids to very different angular divergences. In this case, $\left\langle\theta_{\text {coll }}^{2}\right\rangle \neq 0$ as shown in Fig. 2(c), and we expect $L_{g \text {,coll }}$ to diverge from $L_{g, 3 \mathrm{D}}$.

The parameters listed in Table I are considered for a quantitative comparison of $L_{g, \text { coll }}$ and $L_{g, 3 \mathrm{D}}$ as a function of $\beta_{u}$, in Fig. 3. The FEL wavelength and the emittance growth were chosen in order to ensure $\left\langle\theta_{\text {coll }}^{2}\right\rangle<\theta_{\text {th }}^{2} / \pi$ over the entire range of $\beta_{u}$. The Xie-defined gain length $L_{g, 3 \mathrm{D}}$ was computed for beam slice normalized emittances of 0.5 (green dashed-dotted line) and $2.3 \mu \mathrm{m}$ (red dashed line); in these cases, the projected emittances coincide with the sliced values, since all slices are well aligned in the phase

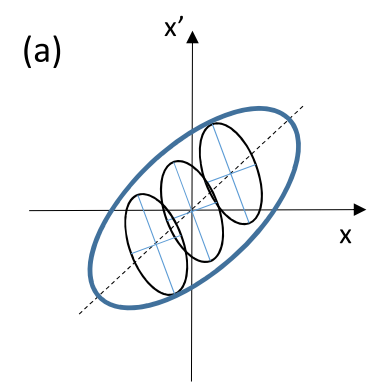

(b)
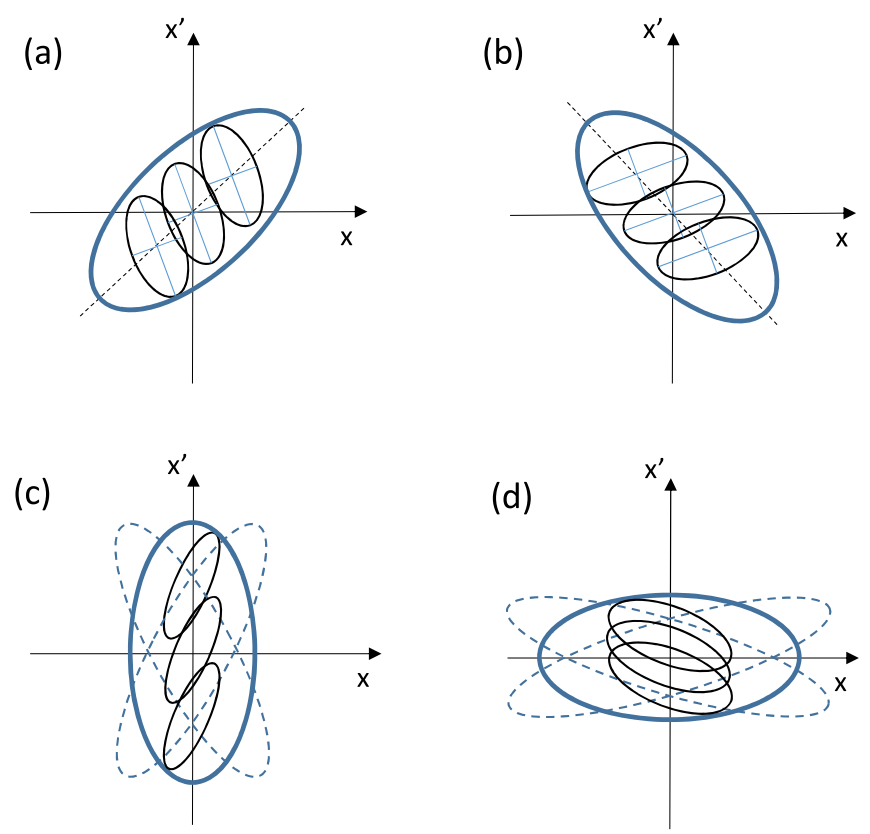

FIG. 2. Mechanism of emittance growth in the transverse phase space, due to kicks by collective effects (cartoon). (a) Two macroslices are displaced along the direction of the kick (dashed line) with respect to an unperturbed macroslice (inner centered ellipse). The projected emittance has grown (outer ellipse). (b) The same as in (a), after $\pi / 2$ betatron phase advance. The area of the outer ellipse remains constant after the kick. (c) The beam is matched at the entrance of the undulator to some design Twiss parameters. The optics is smooth in a way that Twiss parameters $\beta$ and $\alpha$ vary little along the undulator (dashed outer ellipses). Since $\beta_{u}$ is small, the macroslices are largely dispersed in angular divergence; that is, $\left\langle\theta_{\text {coll }}^{2}\right\rangle \neq 0$ (solid line ellipse). (d) The same as in (c), but with $\beta_{u}$ large. The macroslices largely overlap in angular divergence; that is, $\left\langle\theta_{\text {coll }}^{2}\right\rangle \rightarrow 0$ (solid line ellipse).

space. $L_{g \text {,coll }}$ instead, was computed for a beam slice normalized emittance of 0.5 and $2.3 \mu \mathrm{m}$ normalized projected emittance (blue solid line). The latter is determined by the misalignment of the bunch slices in the phase space. The analytical predictions are in agreement with the simulation results obtained with the Genesis code [16], over the entire range of $\beta_{u}$ considered (symbols), thus demonstrating the validity of the proposed gain length

TABLE I. Parameters for the SASE FEL used to compare $L_{g \text {,coll }}$ [Eq. (2)] and $L_{g, 3 \mathrm{D}}$ [4], as a function of $\beta_{u}$ in the undulator.

\begin{tabular}{lcc}
\hline \hline Parameter & Value & Unit \\
\hline Energy & 1.8 & $\mathrm{GeV}$ \\
Peak current & 3.0 & $\mathrm{kA}$ \\
Norm. transv. emittance at the injector, rms & 0.5 & $\mu \mathrm{m} \mathrm{rad}$ \\
Norm. transv. emittance at the undulator, rms & 2.3 & $\mu \mathrm{m} \mathrm{rad}$ \\
Undulator parameter (planar undulator), K & $\sqrt{2}$ & \\
Undulator period & 20 & $\mathrm{~mm}$ \\
FEL parameter, 1D (for $\left.\beta_{u}=10 \mathrm{~m}\right)$ & 0.1 & $\%$ \\
Fundamental wavelength & 1.6 & $\mathrm{~nm}$ \\
\hline \hline
\end{tabular}




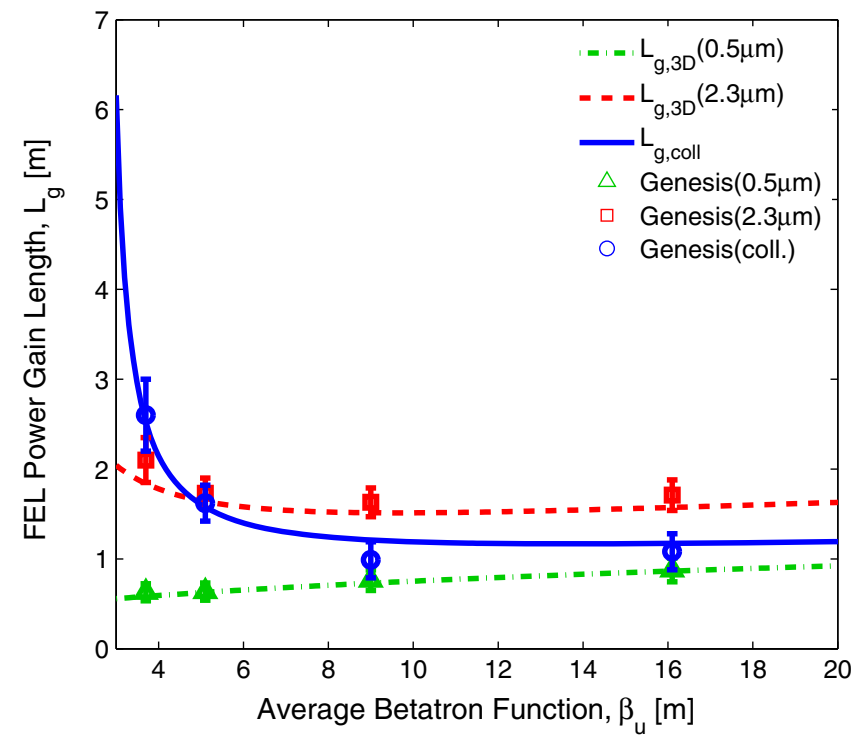

FIG. 3. Gain length as a function of the average betatron function in the undulator, analytical (lines) and from Genesis simulation (symbols), with parameters from Table I. The gain length from simulations fits the FEL power growth along the undulator. Error bars show the maximum variation of the fit value over several runs. For each run used to fit $L_{g, \text { coll }}$ (blue circles), several random distributions of the bunch's macroslices in the transverse phase space were generated. In this case, each distribution (in each transverse plane) corresponds to a normalized projected emittance of $2.3 \mu \mathrm{m}$, while the slice emittance is $0.5 \mu \mathrm{m}$ for all slices. The projected beam size is forced to fit the average betatron function selected for that run. In the two other cases shown in the figure, the projected and the slice normalized emittances coincide, with values 0.5 (green triangles) and $2.3 \mu \mathrm{m}$ (red squares), respectively. The average betatron function in the abscissa is the geometric mean of the horizontal and the vertical $\beta$ averaged along the undulator.

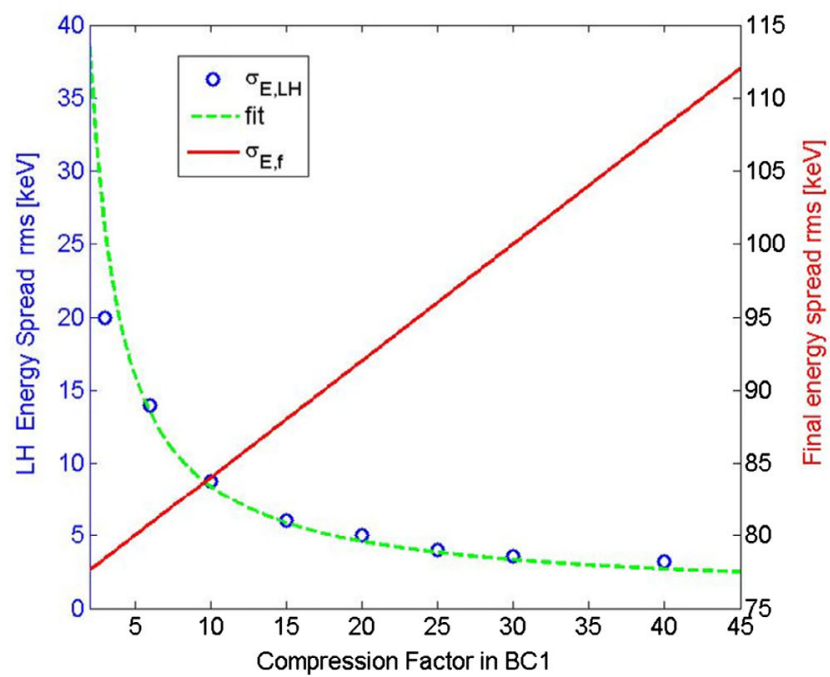

S-BAND LINAC (SL)
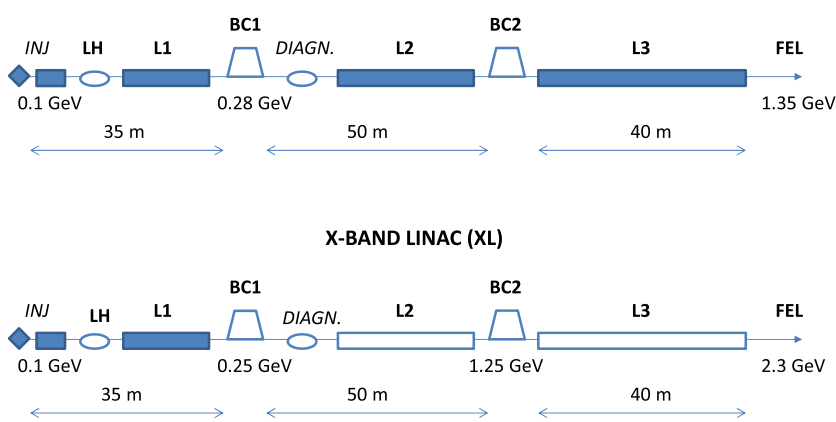

FIG. 5. Sketch of the linacs modeled for the brightness and the gain length study (not to scale). Filled rectangles are for $S$-band $\mathrm{rf}$ structures; empty rectangles are $X$-band. Labels in bold refer to elements modeled in this article.

model and its consistency with the existing 3D theory. Most EUV and $x$-ray FELs, existing and planned, tend to have $\beta_{u}$ small in order to maximize the transverse overlap of electrons and photons in the undulator. Figure 3 suggests that a beam focusing less tight than foreseen for an ideal beam might be more suitable in the presence of a highly diluted projected emittance.

The physics depicted so far applies, in principle, both to SASE and to externally seeded FELs, because, independently from the FEL start-up signal, they both rely on the amplification of undulator radiation through the formation of bunching at the resonance wavelength. In practice, however, in a SASE FEL the entire bunch participates to lasing, while for externally seeded FELs only the seeded portion of the electron bunch is relevant to lasing. In other words, the present analysis applies only to the lasing (seeded) portion of the electron bunch.

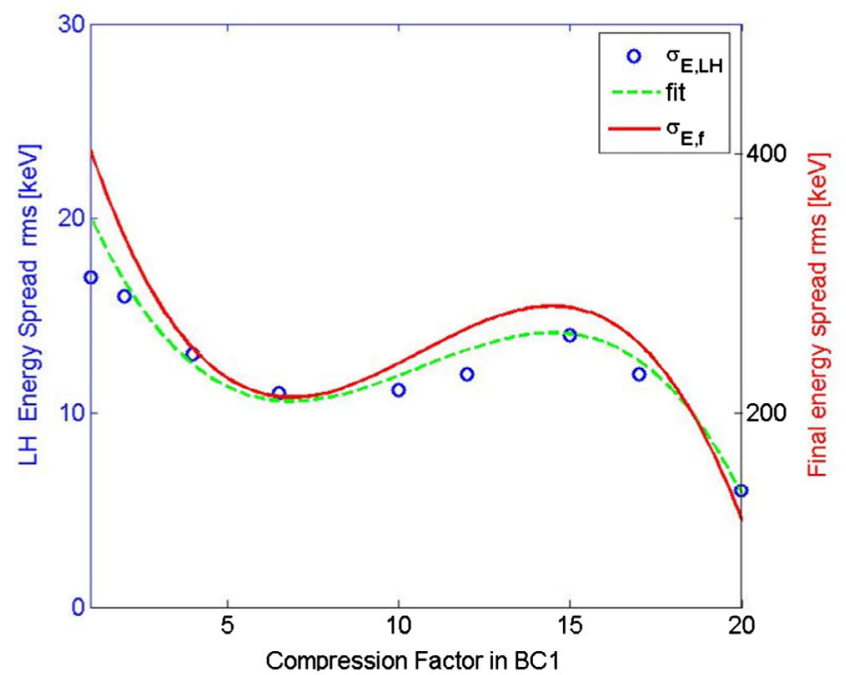

FIG. 4. Laser heater induced rms energy spread and final rms energy spread as a function of the first stage compression factor, for a $500 \mathrm{pC}$ charge, $2.8 \mathrm{ps}$ rms long bunch compressed in one stage (left) and two stages. In the latter, the total compression factor is fixed at 20. Data were evaluated with the analytical model of the MBI given in Refs. $[12,13]$. The energy spread is always meant to be slice. The final energy spread is the energy spread of the heated beam times the total compression factor. 


\section{COLLECTIVE EFFECTS IN THE LONGITUDINAL PLANE}

\section{A. Slice energy spread}

We posit that the final beam slice energy spread is primarily determined by the interplay of MBI and a laser heater $(\mathrm{LH})$ preceding magnetic compressor(s). The minimum amount of beam heating necessary to suppress the instability can be estimated on the basis of analytical $[12,13]$ and numerical tools [17]. For simplicity, we will consider a beam heating strong enough to suppress the MBI, so that the longitudinal emittance is preserved. In this case, the final slice energy spread is given by its value before compression times the total compression factor $C$ :

$$
\sigma_{\delta, f} \approx C \frac{\sigma_{E, i}}{E_{f}} \equiv C \frac{\sqrt{\sigma_{E, 0}^{2}+\sigma_{E, \mathrm{LH}}^{2}}}{E_{f}},
$$

where $E_{f}$ is the final beam energy, $\sigma_{E, 0}$ the slice rms energy spread out of the injector (typically in the $1-3 \mathrm{keV}$

TABLE II. Reference electron beam, accelerator, and FEL parameters for the SL and the XL systems.

\begin{tabular}{|c|c|c|c|c|}
\hline Parameter & Symbol & $S$ band & $X$ band & Units \\
\hline Charge & $Q$ & 500 & 50 & $\mathrm{pC}$ \\
\hline Initial bunch duration, rms & $\sigma_{t 0}$ & 2.8 & 1.6 & ps \\
\hline Initial peak current & $I_{0}$ & 50 & 10 & A \\
\hline Initial norm. transv. emittance, rms & $\varepsilon_{n, 0}$ & 1 & 0.3 & $\mu \mathrm{m} \mathrm{rad}$ \\
\hline Initial uncorr. energy spread, rms & $\sigma_{E, 0}$ & 2 & 2 & $\mathrm{keV}$ \\
\hline LH-induced energy spread, rms & $\sigma_{E, \mathrm{LH}}$ & 8 & 5 & $\mathrm{keV}$ \\
\hline Linac1 rf active length & $L 1$ & 18 & 18 & $\mathrm{~m}$ \\
\hline Linac1 number of structures & & 4 & 4 & \\
\hline Linac1 average acc. gradient & $G_{1}$ & 12 & 10 & $\mathrm{MV} / \mathrm{m}$ \\
\hline Linac1 transv. wakefield amplitude & $W_{\perp, 1}$ & $1 \times 10^{16}$ & $1 \times 10^{16}$ & $\mathrm{~V} / \mathrm{C} / \mathrm{m}^{2}$ \\
\hline Linac2 $\mathrm{rf}$ active length & $L 2$ & 26 & 30 & $\mathrm{~m}$ \\
\hline Linac2 number of structures & & 5 & 60 & \\
\hline Linac2 transv. wakefield amplitude & $W_{\perp, 2}$ & $8 \times 10^{16}$ & $17 \times 10^{16}$ & $\mathrm{~V} / \mathrm{C} / \mathrm{m}^{2}$ \\
\hline Linac2 average acc. gradient & $G_{2}$ & 17 & 35 & $\mathrm{MV} / \mathrm{m}$ \\
\hline Linac3 rf active length & $L 3$ & 30 & 30 & $\mathrm{~m}$ \\
\hline Linac3 number of structures & & 5 & 60 & \\
\hline Linac3 average acc. gradient & $G_{3}$ & 24 & 35 & $\mathrm{MV} / \mathrm{m}$ \\
\hline Linac3 transv. wakefield amplitude & $W_{\perp, 3}$ & $17 \times 10^{16}$ & $17 \times 10^{16}$ & $\mathrm{~V} / \mathrm{C} / \mathrm{m}^{2}$ \\
\hline Linac1 average FODO length & $L_{\mathrm{FODO}, 1}$ & 9 & 9 & $\mathrm{~m}$ \\
\hline Linac1 average betatron function & $\left\langle\beta_{1}\right\rangle$ & 35 & 35 & $\mathrm{~m}$ \\
\hline Linac1 transverse misalignment, $\mathrm{rms}$ & $\Delta_{1}$ & 100 & 100 & $\mu \mathrm{m}$ \\
\hline Linac2 average FODO length & $L_{\mathrm{FODO}, 2}$ & 11 & 6 & $\mathrm{~m}$ \\
\hline Linac2 average betatron function & $\left\langle\beta_{2}\right\rangle$ & 25 & 25 & $\mathrm{~m}$ \\
\hline Linac2 transverse misalignment, $\mathrm{rms}$ & $\Delta_{2}$ & 100 & 100 & $\mu \mathrm{m}$ \\
\hline Linac3 average FODO length & $L_{\mathrm{FODO}, 3}$ & 12 & 6 & $\mathrm{~m}$ \\
\hline Linac3 average betatron function & $\left\langle\beta_{3}\right\rangle$ & 20 & 20 & $\mathrm{~m}$ \\
\hline Linac3 transverse misalignment, $\mathrm{rms}$ & $\Delta_{3}$ & 100 & 100 & $\mu \mathrm{m}$ \\
\hline$B C 1 R_{56}$ & $R_{56,1}$ & -41 & -41 & $\mathrm{~mm}$ \\
\hline$B C 1$ linear compression factor & $C 1$ & 10 & 10 & \\
\hline$B C 1$ minimum horiz. betatron function & $\beta_{1}$ & 5 & 5 & $\mathrm{~m}$ \\
\hline$B C 2 R_{56}$ & $R_{56,2}$ & 0 & -46 & $\mathrm{~mm}$ \\
\hline$B C 2$ linear compression factor & $C 2$ & 1 & 8 & \\
\hline$B C 2$ minimum horiz. betatron function & $\beta_{2}$ & 5 & 5 & $\mathrm{~m}$ \\
\hline Initial 6D norm. brightness & $B_{n, 0}$ & 25 & 55 & $\mathrm{~A} / \mu \mathrm{m}^{2} / \mathrm{keV}$ \\
\hline Final 6D norm. brightness (perturbed) & $B_{n, f}^{n, 0}$ & 1 & 5 & $\mathrm{~A} / \mu \mathrm{m}^{2} / \mathrm{keV}$ \\
\hline Undulator magnetic period & $\lambda_{u}$ & 55 & 35 & $\mathrm{~mm}$ \\
\hline Undulator parameter & $K$ & 1.25 & 0.70 & \\
\hline Polarization & & Circular & Circular & \\
\hline FEL fundamental wavelength & $\lambda$ & 10 & 1.3 & $\mathrm{~nm}$ \\
\hline Undulator average betatron function & $\beta_{u}$ & 8 & 8 & $\mathrm{~m}$ \\
\hline FEL parameter, $1 \mathrm{D}$ & $\rho$ & $1.5 \times 10^{-3}$ & $1.5 \times 10^{-3}$ & \\
\hline Power gain length, $1 \mathrm{D}$ & $L_{g, 1 \mathrm{D}}$ & 1.7 & 1.1 & $\mathrm{~m}$ \\
\hline
\end{tabular}


range [18]) and $\sigma_{E, \mathrm{LH}}$ that induced by the LH (typically above $5 \mathrm{keV}$ ).

In practice, it is desirable to keep $\sigma_{E, \mathrm{LH}}$ to the smallest value that still suppresses microbunching. In this case, $B_{n, 6 \mathrm{D}}$ depends on the compression factor: $B_{n, 6 \mathrm{D}}=$ $B_{n, 6 \mathrm{D}}\left[\sigma_{E, \mathrm{LH}}(C)\right]=B_{n, 6 \mathrm{D}}(C)$. We assess such dependence by using the analytical model of MBI developed in Refs. $[12,13]$. The analytical prediction for the LH-induced and the final energy spread is shown in Fig. 4 for a realistic machine configuration whose details are given in Sec. IV. We studied both one-stage and two-stage compression schemes. The compression factor is scanned by varying the magnetic compressor bending angle, for the same linear energy chirp (i.e., the $z$-correlated fractional energy spread over the bunch length) at the chicane entrance. In the twostage scheme, the total compression factor was kept constant, while the local compression strengths (respectively, $C 1$ and $C 2$ for the first and the second stage) were unbalanced. In Fig. 4, $\sigma_{E, \mathrm{LH}}$ is the minimum energy spread required to suppress MBI that is when the instability gain at the linac end approaches unity. A fit of the analytical data of the LH-induced slice energy spread, also shown in Fig. 4, provides the following laws that will be used in the rest of this article for the computation of $B_{n, 6 \mathrm{D}}$ :

$$
\begin{aligned}
\tilde{\sigma}_{E, \mathrm{LH}}^{1-\text { stage }} & =\left(\frac{76}{C 1}+3.2\right) \mathrm{keV}, \\
\tilde{\sigma}_{E, \mathrm{LH}}^{2 \text {-stage }}= & {\left[-0.016(C 1-9)^{3}+0.08(C 1-13)^{2}\right.} \\
& +0.12(C 1-19)+1] \mathrm{keV}, \quad C 1 \cdot C 2 \equiv 20 .
\end{aligned}
$$

\section{B. Bunch length compression}

In the one-stage compression case depicted in Fig. 4, the LH-induced energy spread is inversely proportional to $C 1$,

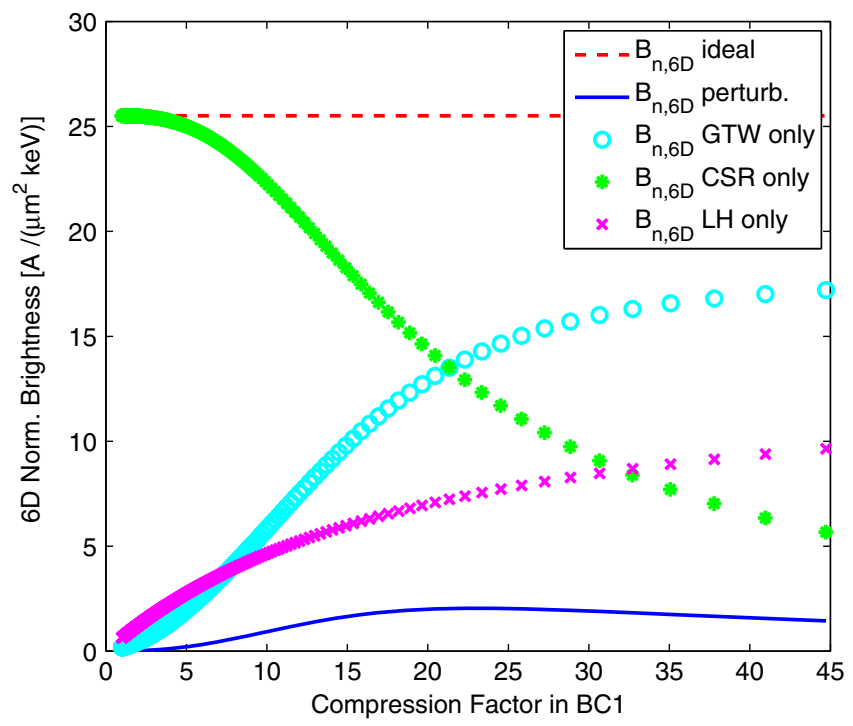

because the stronger compression suppresses the instability via energy Landau damping [see Eq. (4)]. In the two-stage compression, instead, $\sigma_{E, \mathrm{LH}}$ is the result of a balance between strong compression in $B C 1$, which favors energy Landau damping, and high peak current propagating from $B C 1$ to $B C 2$, which drives the instability gain. As the compression in $B C 2$ approaches unity $(C 1$ approaching 20 in the right plot), energy Landau damping in $B C 1$ takes over and we observe a roll-off of $\sigma_{E, \mathrm{LH}}$.

In our model, the rf phase and voltage of some accelerating structures upstream of each compressor are tuned to keep the beam mean energy and the energy chirp fixed. A maximum $2 \%$ rms energy spread is allowed at $B C 1$ and $1 \%$ at $B C 2$. These energy spread levels are typical maxima compatible with magnetic field quality in the compressors' bending magnets in order to avoid chromatic transverse emittance dilution. The highest acceptable correlated energy spread brings about the minimum bending angle for a given compression factor without beam quality disruption [19,20]. Other approaches for scanning the compression strength might be equally valid. For example, the beam energy chirp may be varied while keeping the bending angle in the compressor fixed. This may lead to different laws for $\sigma_{E, \mathrm{LH}}(C)$ that, nevertheless, may still be computable with the proposed procedure.

\section{ELECTRON BEAM BRIGHTNESS}

\section{A. Definition of the 6D normalized brightness}

The $6 \mathrm{D}$ normalized electron beam brightness $B_{n, 6 \mathrm{D}}$ is defined as the total bunch charge over the product of the horizontal, vertical, and longitudinal rms normalized projected emittance. The normalized longitudinal emittance is the product of bunch length and absolute energy spread. All three emittances are invariant under acceleration and linear

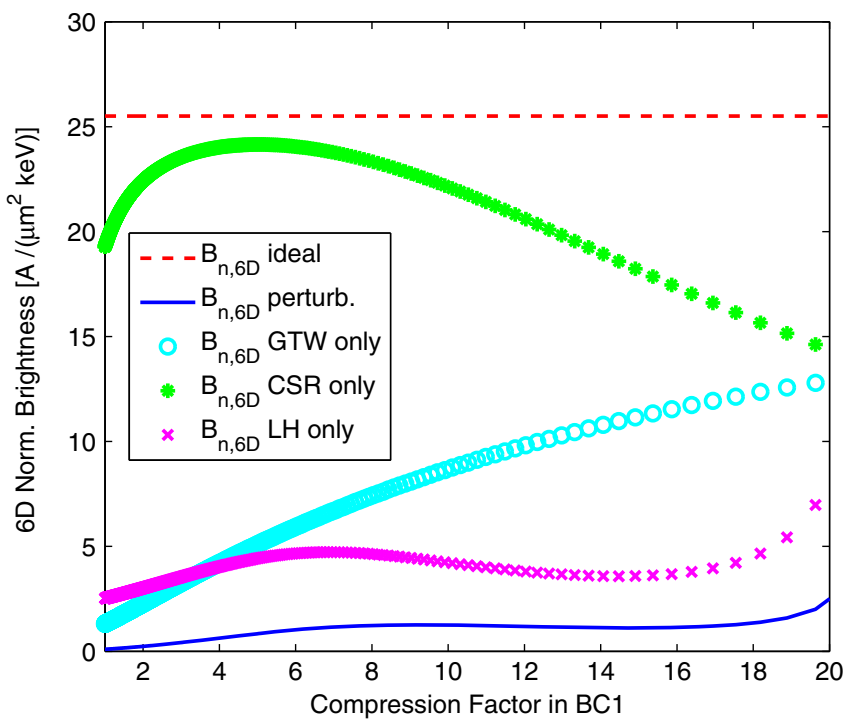

FIG. 6. Analytical estimate of $B_{n, 6 \mathrm{D}}$ at the end of SL (see Fig. 3) for the one-stage (left) and two-stage compression, as a function of the compression factor in $B C 1$. In the two-stage, the total compression factor is fixed to 20 . 
bunch length compression, and in the absence of collective effects. The bunch length compression process in magnetic chicane(s) is assumed to be linearized by a high harmonic rf cavity [21,22]. In the following, we refer to Refs. [10,14] and references therein for modeling the GTW and the CSR effect on the transverse emittance. In particular, Bane's model $[23,24]$ of the transverse wake function is adopted, while the emittance growth due to misaligned accelerating structures is analytically estimated like in Ref. [25]. Control of the CSR-induced emittance growth via optics manipulation in a chicane for magnetic compression is taken from Ref. [26].

We assume the CSR and the GTW kicks to be uncorrelated; that is, they add in quadrature. The final normalized (horizontal) emittance subjected to CSR in $n$ consecutive compression stages and to GTW in $m$ linac sections is provided by the determinant of the "sigma matrix" computed at the linac end:

$\varepsilon_{n, f} \cong \varepsilon_{n, 0} \sqrt{\left(1+P_{\mathrm{CSR}}^{B C 1}\right)\left(1+P_{\mathrm{CSR}}^{B C 2}\right) \ldots\left(1+P_{\mathrm{CSR}}^{B C n}\right)\left(1+P_{\mathrm{GTW}}^{L 1}\right)\left(1+P_{\mathrm{GTW}}^{L 2}\right) \ldots\left(1+P_{\mathrm{GTW}}^{L m}\right)} \approx \varepsilon_{n, 0} \sqrt{1+\sum_{i=1}^{n} P_{\mathrm{CSR}}^{i}+\sum_{j=1}^{m} P_{\mathrm{GTW}}^{j}}$.

The last equality in Eq. (5) was used in Ref. [10] and is valid under the assumption that the individual perturbations to the initial normalized emittance are small: $P_{\text {CSR }}^{j}, P_{\text {GTW }}^{j} \ll 1 \forall i, j$. In this article, the first more general equality in Eq. (5) is used. The identity of Eqs. (5) and (1) allows us to compute $\left\langle\theta_{\text {coll }}^{2}\right\rangle$ once $\beta_{u}$ and $\varepsilon_{n, f}$ are known. The explicit expression of $P_{\mathrm{CSR}}^{i}, P_{\mathrm{GTW}}^{j}$ is given in Appendixes A and B of this article.

We further simplify the expression of the final emittances by introducing an effective degradation factor $\zeta \geq 1$ in each plane of the particle motion: $\varepsilon_{n x, f}=\zeta_{x} \gamma_{0} \varepsilon_{x, 0}$, $\varepsilon_{n y, f}=\zeta_{y} \gamma_{0} \varepsilon_{y, 0}$, and $\varepsilon_{n z, f}=\sigma_{z, f} \sigma_{E, f}=\sigma_{z, 0} \sigma_{E, i}=\zeta_{z} \varepsilon_{z, 0}$, with obvious notations. $\zeta_{x}$ and $\zeta_{y}$ express the combined effects of CSR and GTW in the transverse planes; $\zeta_{z}=$ $\sigma_{E, i} / \sigma_{E, 0}$ is the ratio of the slice energy spread before compression (including that induced by the LH) and the slice energy spread produced at the injector [see Eq. (3)]. We are now able to relate $B_{n, 6 \mathrm{D}}$ at the undulator to that at the linac injection, $B_{n, 0}$ :

$B_{n, 6 \mathrm{D}} \equiv \frac{Q}{\varepsilon_{n x, f} \varepsilon_{n y, f} \varepsilon_{n z, f}}=\frac{Q}{\zeta_{x} \zeta_{y} \zeta_{z} \gamma_{0}^{2} \varepsilon_{x, 0} \varepsilon_{y, 0} \sigma_{z, 0} \sigma_{E, 0}}=\frac{B_{n, 0}}{\zeta_{x} \zeta_{y} \zeta_{z}}$

where $Q$ is the total bunch charge. In the ideal case of vanishing collective effects, $P_{\mathrm{CSR}}^{i}, P_{\mathrm{GTW}}^{j} \rightarrow 0$ in Eq. (5) and $\zeta_{x}, \zeta_{y}, \zeta_{z} \rightarrow 1$ in Eq. (6), so that the 6D normalized brightness is preserved at the injector level under acceleration and linear compression.

It is worth noticing that in our model the slice emittance is expected to grow via GTW and CSR only when the bunch duration is strongly compressed, e.g., down to the scale of a few FEL cooperation lengths. In this case, the effect of slice emittance growth on the FEL gain length can be modeled via $L_{g, 3 \mathrm{D}}$ in Eq. (2). Moreover, our model does not explicitly consider any beam-based alignment procedure that could alleviate the effect of GTW. Such a
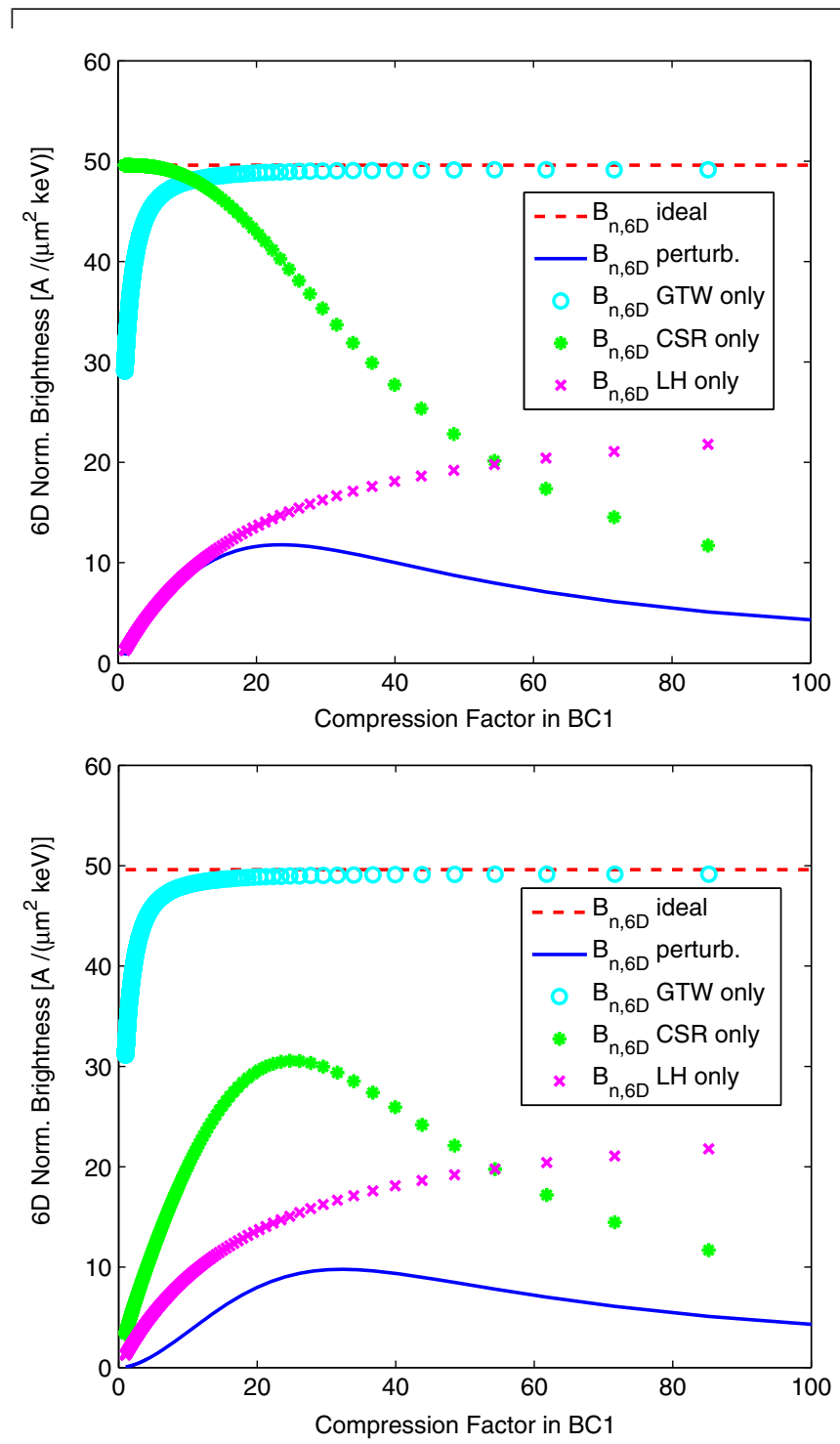

FIG. 7. Analytical estimate of $B_{n, 6 \mathrm{D}}$ at the end of XL (see Fig. 3) for the one-stage (left) and two-stage compression, as a function of the compression factor in $B C 1$. In the two-stage scheme, the total compression factor is fixed to 100 . 

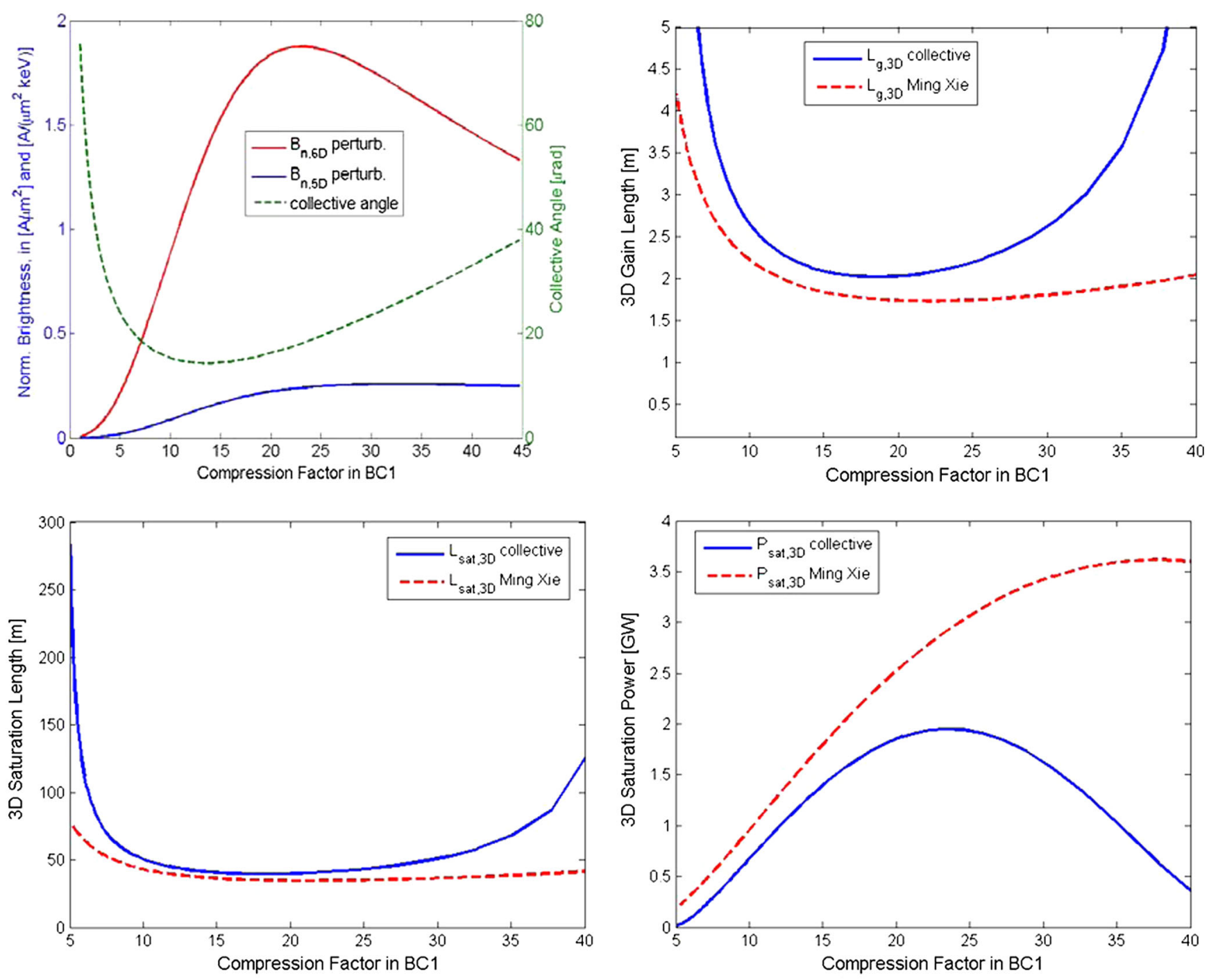

FIG. 8. The final 5D and 6D normalized brightness and the collective angle [Eq. (2)] are evaluated vs the compression factor in $B C 1$ (top left panel), for a $500 \mathrm{pC}$ bunch charge compressed in SL with one stage. The 3D gain length (top right), the 3D SASE saturation length (bottom left), and the 3D SASE saturation power are evaluated in the Xie sense and in the presence of collective effects, respectively.

scenario, however, may be taken into account by considering an effective linac-to-beam misalignment (see Appendix B) smaller than the one provided by static machine alignment. Finally, we neglect the contribution of the $z$-correlated energy spread to the slice energy spread. In practice, we assume that the bunch's longitudinal phase space is controlled, e.g., like in Ref. [27]. More in general, we assume that the final rms fractional energy spread evaluated over the bunch slice length is smaller than the FEL energy-normalized bandwidth $\rho$ (also named the "FEL parameter") [6].

\section{B. Linac modeling}

Two single-pass linacs, one for EUV and the other for $\mathrm{x}$-ray FEL, are introduced in Fig. 5 and in Table II. For both linacs, we investigated two options: one-stage compression at low energy and two-stage compression with fixed total compression factor $C=C 1 \times C 2$. We then looked at $B_{n, 6 \mathrm{D}}$ vs $C 1$ to identify the compression scheme that maximizes the beam brightness for a given final peak current (1 kA). That compression scheme was then used in the remainder of this article to compare the FEL 3D gain length and other related FEL parameters in the presence of collective effects to those predicted by Xie, over a wide range of compression strengths. Finally, we selected the compression factor that minimizes $L_{g, \text { coll }}$ and studied its sensitivity, as well as that of $B_{n, 6 \mathrm{D}}$, to the linac-to-beam misalignment and the optics in the compressor.

The first linac layout, named SL, reflects the existing $S$-band FERMI beam line [28]: it accelerates a beam charge of $500 \mathrm{pC}$ over approximately $1 \mathrm{ps}$ full width final bunch duration. The second linac, named XL, is inspired by a 

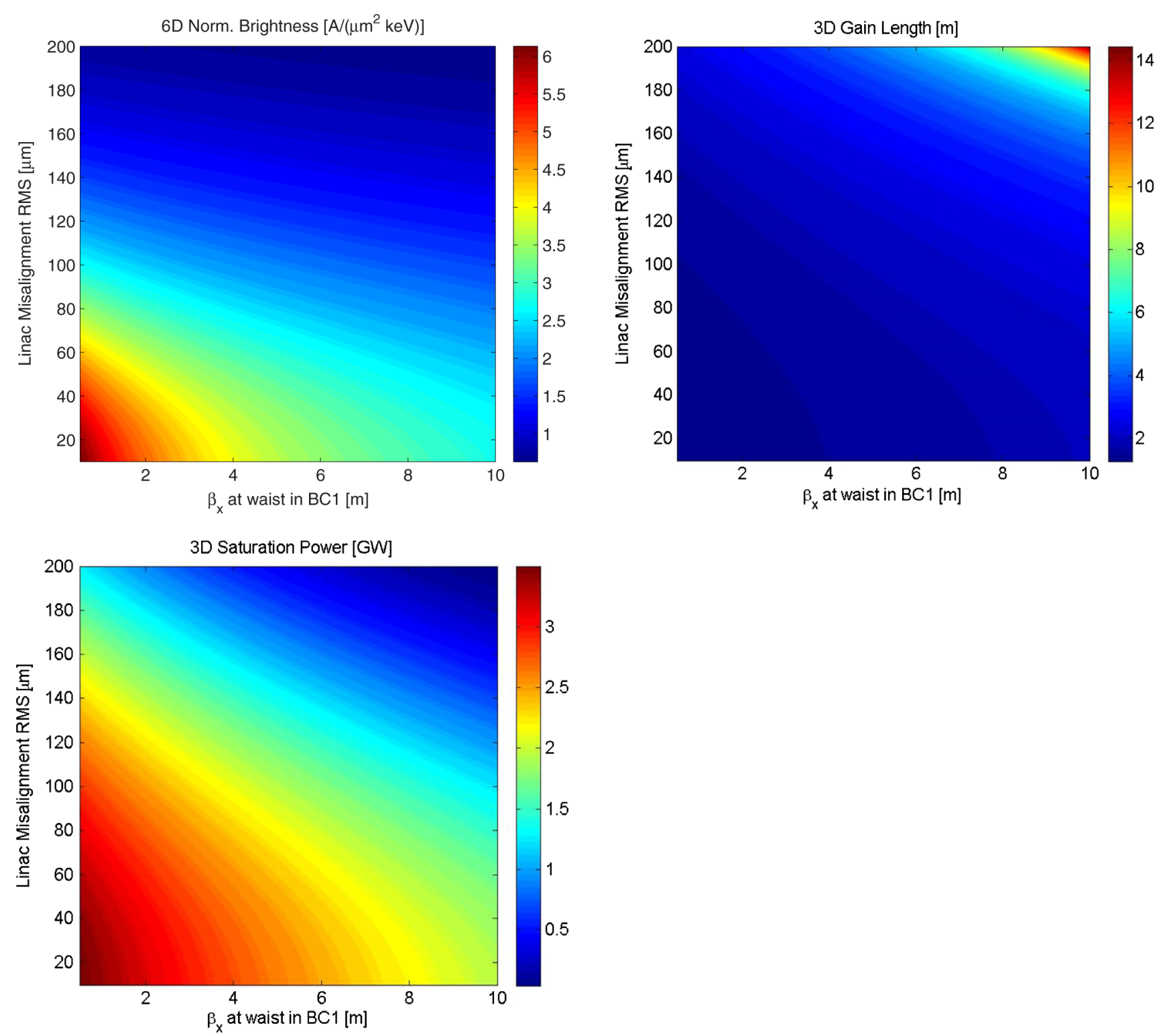

FIG. 9. Contour plot of $B_{n, 6 \mathrm{D}}$ (top left), $L_{g, \text { coll }}$ (top right), and $P_{\text {sat,coll }}$ as a function of the rms linac-to-beam misalignment and of the minimum betatron function in $B C 1$. A $500 \mathrm{pC}$ bunch charge, compressed in one stage by a factor of 18 in the SL linac, was considered. The final peak current is $0.9 \mathrm{kA}$.

Bartolini's study [29] for a high repetition rate $X$-band FEL driver. It is meant to accelerate a lower bunch charge of $50 \mathrm{pC}$, strongly compressed to reach a high final peak current. It may even be thought as an upgraded SL based upon $X$-band technology (see, e.g., [30]).

Figure 6 shows the behavior of $B_{n, 6 \mathrm{D}}$ in SL when $C 1$ is varied, in the one-stage (left) and two-stage compression. The latter is run with $C=C 1 \times C 2=20$. As expected, the unperturbed $B_{n, 6 \mathrm{D}}$ is independent of $C$ (dashed line). When collective effects are included, CSR suppresses $B_{n, 6 \mathrm{D}}$ at large $C 1$ (shorter bunches emit a stronger CSR field, which leads to larger $\varepsilon_{n x}$ growth), while GTW is stronger at small $C 1$. A local maximum for $B_{n, 6 \mathrm{D}}$ is then generated (solid line) because of the opposite dependence of CSR and GTW on the compression strength. As for the longitudinal emittance, $B_{n, 6 \mathrm{D}}$ depends on $C 1$ like the inverse of Eq. (4), which explains the increase of brightness at large $C 1$. Figure 6 suggests that, for a high charge bunch moderately compressed $(C=20)$ in SL to reach, e.g., $1 \mathrm{kA}$, the one-stage compression promises a higher $B_{n, 6 \mathrm{D}}$ than the two-stage. For this reason, the one-stage compression will be chosen for the study of the FEL gain length in the next section. Figure 7 shows that in the XL the effect of the GTW is greatly suppressed by the low charge and the short bunch duration, so that the maximum of $B_{n, 6 \mathrm{D}}$ is mainly determined by the opposing dependences of the CSR strength and of the slice energy spread on $C 1$. The evaluation of the LH-induced energy spread vs $C 1$ in $\mathrm{XL}$ 

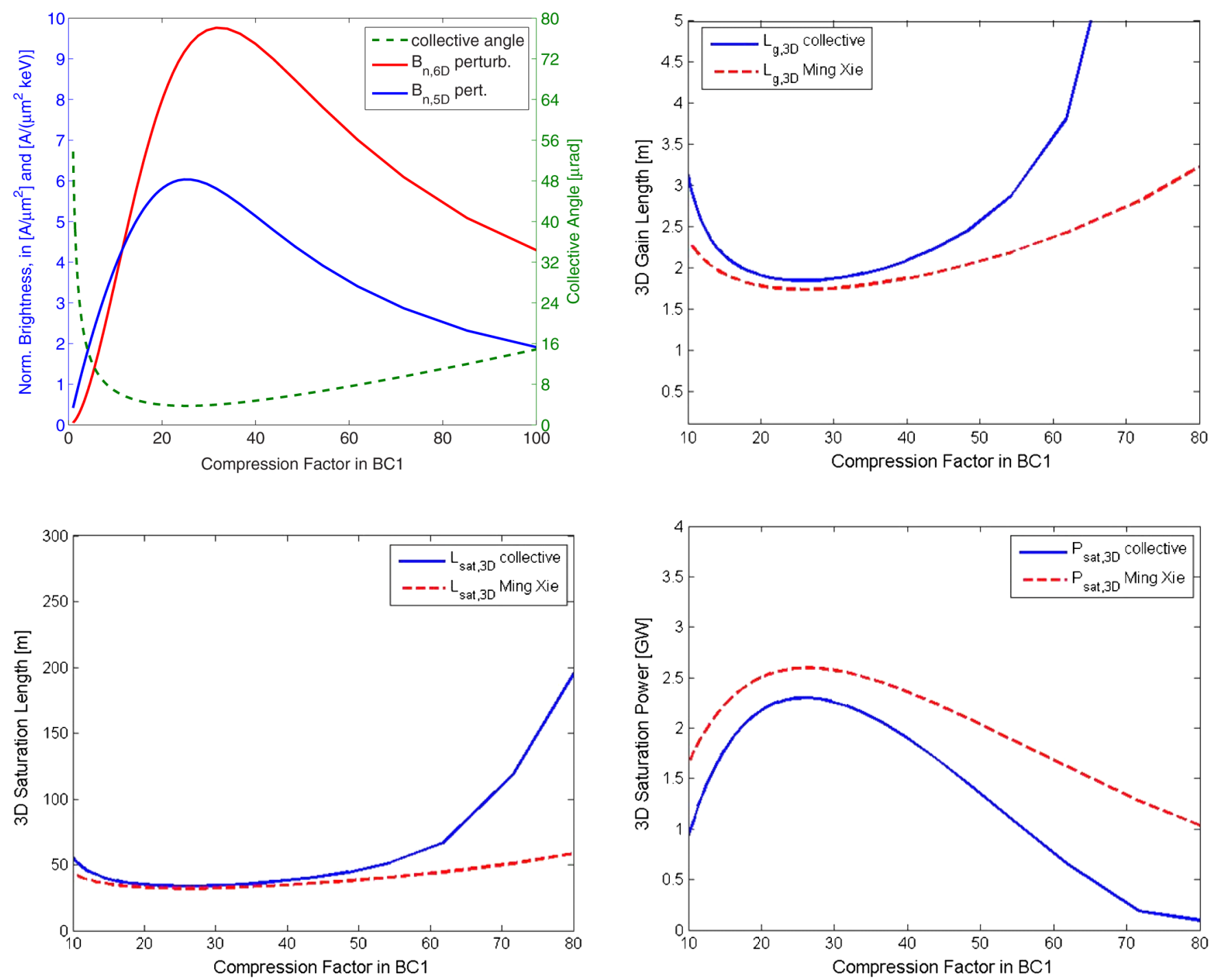

FIG. 10. Final 5D and 6D normalized brightness and collective angle [Eq. (2)] vs compression factor in $B C 1$ (top left panel) for a $50 \mathrm{pC}$ bunch charge accelerated in XL with two-stage compression; the total compression factor is set at 100 and the final peak current is $1 \mathrm{kA}$. The 3D gain length (top right), 3D SASE saturation length (bottom left), and 3D SASE saturation power are evaluated in the Xie sense and in the presence of collective effects, respectively.

follows laws which are similar to those depicted in Eq. (4) and not reported here for the sake of brevity. In XL, the two-stage compression promises a slightly larger $B_{n, 6 \mathrm{D}}$ than the one-stage, for the same $1 \mathrm{kA}$ final peak current. In this case, the total compression factor is set at 100 .

\section{FEL PERFORMANCE}

\section{1. $S$-band linac with high charge}

We compare in Fig. 8 the Xie-defined FEL 3D output performance with that predicted by our theory, for the SL system with one-stage compression (see Sec. IV). The Xie-defined SASE FEL 3D saturation length is [4] $L_{\text {sat }, 3 \mathrm{D}}^{M . \text { Xie }}=L_{g, 3 \mathrm{D}} \ln \left(\frac{9 P_{\text {sat }, \mathrm{D}}}{P_{\text {noise }}}\right)$, with $P_{\text {sat }, 3 \mathrm{D}} \approx 1.6 \rho E I\left(\frac{L_{g, 1 \mathrm{D}}}{L_{g, 3 \mathrm{D}}}\right)^{2}, I$ the bunch final peak current, $E$ the beam mean energy, and $P_{\text {noise }} \approx \rho^{2} c E / \lambda$. We defined the 3D saturation length in the presence of collective effects in an analogous manner: $L_{\text {sat }, 3 \mathrm{D}}^{\text {coll }}=L_{g, \text { coll }} \ln \left(\frac{9 P_{\text {sat, coll }}}{P_{\text {noise }}}\right)$, where $P_{\text {sat, coll }} \approx 1.6 \rho E I\left(\frac{L_{g, 1 \mathrm{D}}}{L_{g, \text { coll }}}\right)^{2}$. We note that a range of $C 1$ can be found where the SASE gain length and saturation length do not vary appreciably. In contrast, the SASE power at saturation shows a larger sensitivity to $C 1$, just as $B_{n, 6 \mathrm{D}}$ does. For completeness, the values of the collective angle and $B_{n, 5 \mathrm{D}}$ are also shown as a function of $C 1 . B_{n, 5 \mathrm{D}}$ is the transverse beam brightness, namely, $B_{n, 6 \mathrm{D}}$ times $\sigma_{E, f}$.

We then focus on the region around the point of minimum gain length in Fig. $8(C 1=18)$ for the study of the sensitivity of $B_{n, 6 \mathrm{D}}, L_{g, \text { coll }}$, and $P_{\text {sat,coll }}$ to the linac-tobeam misalignment (which drives the GTW instability; see Appendix B) and the betatron function in $B C 1$ (which 

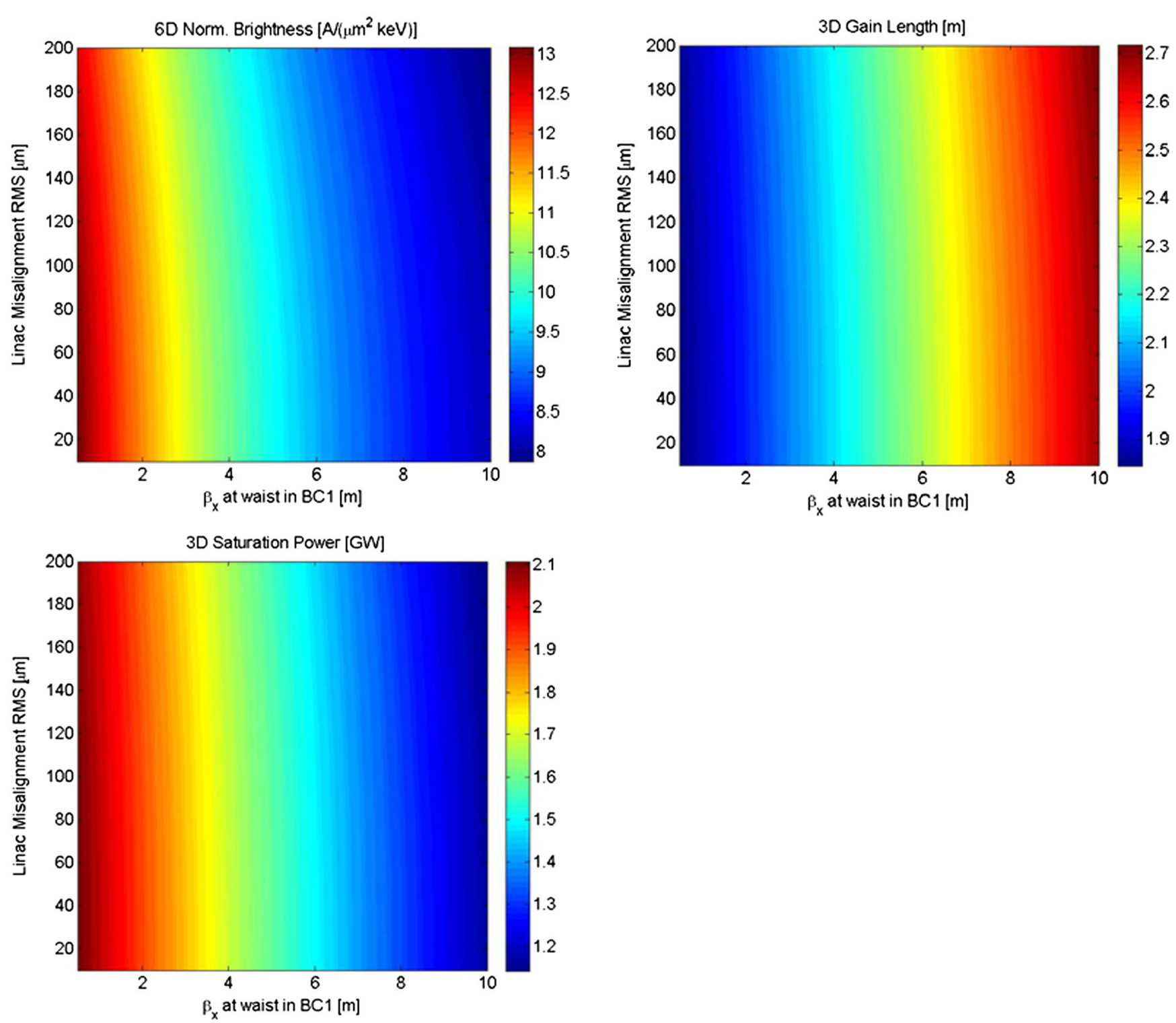

FIG. 11. Contour plot of $B_{n, 6 \mathrm{D}}$ (top left), $L_{g, \text { coll }}$ (top right), and $P_{\text {sat,coll }}$ as a function of the rms linac-to-beam misalignment and the minimum betatron function in $B C 1$, for a $50 \mathrm{pC}$ bunch charge, compressed by a factor $C=25 \times 4$ in the XL linac. The final peak current is $1 \mathrm{kA}$.

controls the CSR-induced emittance growth; see Appendix A). The results are shown in Fig. 9.

\section{2. $X$-band linac with low charge}

We repeat the previous study for the XL system with the two-stage compression, where $C 1$ is varied and the total compression factor is fixed to 100 . The comparison of the Xie-defined FEL performance with ours is given in Fig. 10. The point of minimum gain length is identified in Fig. 10 $(C 1=25)$, and, around it, the linac-to-beam misalignment and the betatron function in $B C 1$ are varied to study the sensitivity of $B_{n, 6 \mathrm{D}}$ and of the FEL performance to these parameters. The sensitivity study for the XL-driven FEL is shown in Fig. 11.

\section{CONCLUSIONS AND FINAL REMARKS}

We have extended the existing analytical models for the estimation of the electron beam brightness [10] and of FEL properties [4,5,8] — gain length, saturation length, and power at saturation of a SASE FEL-by including the collective effects in the driving linac. In particular, we took into account the increase of slice energy spread when MBI is suppressed with a $\mathrm{LH}$ and the consequences of timeaveraged (along the bunch) transverse tilts of electron bunch's macroslices due to CSR in bunch compressors and GTW in accelerating structures. Two major findings follow from the proposed model: (1) We showed that the degradation of the beam transverse projected emittance affects the FEL performance even though the slice 
emittance is preserved. Our analytical finding for the 3D gain length in the presence of collective effects, i.e., Eq. (2), is in agreement with Genesis simulation results within $5 \%-15 \%$ of the gain length, over the wide range of $\beta_{u}$ considered (see Fig. 3). The residual analysis vs simulation discrepancy may originate from the lack of several approximations in the Genesis runs, which are instead part of our theory: the asymmetry of horizontal and vertical betatron function (at large $\beta_{u}$ ), whereas our model assumes perfect symmetry; the effect of multiple angular kicks on the offset bunch's macroslices by quadrupole magnets, which are neglected in our model [8]; the power gain computed from time-dependent simulations instead of the steady-state approximation (single longitudinal FEL mode), which is part of Tanaka's model $[8,9]$. (2) The enlargement of the FEL power gain length due to a dilution of the projected emittance can be counteracted by a relatively large average betatron function in the undulator line. The optimum value of the average betatron function (i.e., corresponding to the minimum gain length) turns out to be closer to the value dictated by the projected emittance with respect to that associated to the slice emittance.

Our model was then compared with that by Xie [4], with the following quantitative findings: (i) A deterioration of the FEL performance with respect to Xie's model [4] is observed when collective effects are included. For the cases considered here, a discrepancy of $\sim 15 \%$ between $L_{g, 3 \mathrm{D}}$ and $L_{g \text {,coll }}$ is observed around the point of minimum gain length and a much larger discrepancy at very small and very large values of $C 1$. (ii) The collective effects halve the "good" range of $C 1$ over which the gain length and the saturation length are a little sensitive (i.e., vary less than $10 \%$ ) to the compression factor. (iii) The SASE power at saturation in the Xie sense is reduced by the collective effects by a factor up to 3 in the $C 1$ range considered. The proposed analysis does not pretend to replace sophisticated FEL codes like Genesis 1.3 [16] and Ginger [31]. Our analysis may be useful for an initial exploration of the design parameters of a high brightness linac-driven FEL and of the magnetic lattice in the undulator line. As a matter of fact, the analytical model described in this article allowed us to investigate and to optimize, as a practical case study, two different accelerator layouts by inspecting two compression schemes and to scan the FEL properties vs the compression strength, the linac-to-beam misalignment, and the betatron function in the magnetic compressor. Our study establishes the predominant influence of GTW on $B_{n, 6 \mathrm{D}}$ for a high charge beam driven by an $S$-band linac and that of CSR for a low charge beam in an $X$-band FEL driver. We observed a net dependence of the FEL saturation power on $B_{n, 6 \mathrm{D}}$. We also found that the gain length and the saturation length can be made quite insensitive to the linac-to-beam misalignment (i.e., GTW instability) and to the optics in the compressor (i.e., CSR instability) with a proper choice of the compression scheme and strength.

\section{ACKNOWLEDGMENTS}

The authors are in debt to M. Cornacchia for his encouragement and careful reading of this article and to W. Fawley for stimulating discussions about the dependence of FEL performance on the beam optics. We acknowledge A. Zholents for his suggestion to extend our work to the $6 \mathrm{D}$ brightness and E. Allaria for his advice on FEL physics. This work was funded by the FERMI project of Elettra Sincrotrone Trieste, partially supported by the Ministry of University and Research under Grants No. FIRB-RBAP045JF2 and No. FIRB-RBAP06AWK3.

\section{APPENDIX A: CSR-INDUCED EMITTANCE GROWTH}

CSR-induced emittance growth is primarily due to betatron oscillations of the bunch longitudinal macroslices around different energy-dispersive trajectories. Even in t he case of achromatic lines, the CSR-increased betatron invariant of the macroslice centroid is not recovered as the dispersion function collapses to zero [32]. If we describe the CSR effect in a short magnet with the single-kick approximation, we are allowed to use the sigma matrix formalism to obtain an expression for the final projected emittance at the exit of the $i$ th magnetic compressor [33]:

$$
\begin{aligned}
\varepsilon_{n, f} & \cong \gamma\left[\operatorname{det}\left(\begin{array}{cc}
\varepsilon_{0} \beta+\eta^{2} \sigma_{\delta, \mathrm{CSR}}^{2} & -\varepsilon_{0} \alpha+\eta \eta^{\prime} \sigma_{\delta, \mathrm{CSR}}^{2} \\
-\varepsilon_{0} \alpha+\eta \eta^{\prime} \sigma_{\delta, \mathrm{CSR}}^{2} & \varepsilon_{0} \frac{1+\alpha^{2}}{\beta}+\eta^{\prime 2} \sigma_{\delta, \mathrm{CSR}}^{2}
\end{array}\right)\right]^{1 / 2} \\
& =\varepsilon_{n, 0} \sqrt{1+\frac{\gamma H}{\varepsilon_{n, 0}} \sigma_{\delta, \mathrm{CSR}}^{2}} \equiv \varepsilon_{n, 0} \sqrt{1+P_{\mathrm{CSR}}^{i}}
\end{aligned}
$$

where $\varepsilon_{n, f}$ and $\varepsilon_{n, 0}$ are the normalized rms emittance after and before the CSR kick, respectively, $H=$ $\left[\eta^{2}+\left(\beta \eta^{\prime}+\alpha \eta\right)^{2}\right] / \beta$ in the bending plane, $\eta$ is the energy dispersion function, $\eta^{\prime}$ its first derivative with respect to the curvilinear longitudinal coordinate $s$, and $\sigma_{\delta \text {, CSR }}$ the CSR-induced rms fractional energy spread. For a Gaussian longitudinal charge distribution [34],

$$
\sigma_{\delta, \mathrm{CSR}}=0.2459 \frac{r_{e}}{e} \frac{Q}{\gamma}\left(\frac{l \theta^{2} C^{4}}{\sigma_{z, 0}^{4}}\right)^{1 / 3},
$$

where we introduced the electron classical radius $r_{e}$ and the electron charge $e, l$ the dipole magnet length, $\sigma_{z, 0}$ the initial rms bunch length, and $C$ the linear compression factor, i.e., the ratio of the initial over the final bunch length:

$$
C=\frac{\sigma_{z, 0}}{\sigma_{z, f}}=\frac{1}{1+h R_{56}} \approx \frac{1}{1+\frac{\sigma_{\delta, 0}}{\sigma_{z, 0}} R_{56}} .
$$

Here $\sigma_{\delta, 0}$ is the rms fractional energy spread imparted to the beam by upstream rf off-crest acceleration. The 
longitudinal transport matrix element $R_{56}$ for a four-dipole, achromatic, and symmetric chicane with bending angle $\theta \ll 1$ is $R_{56} \cong-2 \theta^{2}\left(L_{12}+\frac{2}{3} l\right)$, where $L_{12}$ is the drift length between the two outer bending magnets. In our convention, a negative $R_{56}$ compresses the bunch duration if the linearly correlated energy chirp $h$ is positive.

Since $\sigma_{\delta, \text { CSR }}$ is inversely proportional to the bunch length, the transverse CSR effect in a magnetic chicane is dominated by the radiation emission in the second half of the system, where the bunch reaches its shortest duration. Thus, Eq. (A1) prescribes to shrink the $H$ function in the second half of the chicane in order to suppress the CSR-induced emittance growth. When $\theta \ll 1$ and in the presence of a beam waist (minimum of the betatron function) in the bending plane, this prescription reduces to shrink $\beta$ in proximity of the fourth dipole magnet [35].

\section{APPENDIX B: GTW-INDUCED EMITTANCE GROWTH}

The single-bunch transverse wakefield instability [36], which happens when the bunch travels at a certain distance from the linac electric axis, generates a displacement of the trailing particles respect to the bunch head; this displacement is correlated with the longitudinal coordinate along the bunch. The trailing particles start a betatron oscillation around a new dispersive trajectory, therefore increasing their betatron invariant. Such an effect can be removed by finding a "golden" trajectory which makes the wakefield kicks cancel each other [37]. In analogy with the CSR case (see Appendix A), we are allowed to use the sigma matrix formalism to obtain an expression for the final projected emittance at the exit of the $j$ th linac section [10,23]:

$$
\begin{aligned}
\varepsilon_{n, f} & \cong \varepsilon_{n, 0} \sqrt{1+2\left(\frac{\pi r_{e}}{Z_{0} c e}\right)^{2} \frac{Q^{2} \bar{W}_{\perp}^{2} \Delta^{2} L_{\mathrm{FODO}} L_{\mathrm{tot}} \bar{\beta}}{\varepsilon_{n, 0} \sqrt{\gamma_{0} \gamma_{f}}}} \\
& \equiv \varepsilon_{n, 0} \sqrt{1+P_{\mathrm{GTW}}^{j}},
\end{aligned}
$$

where $Z_{0}=377 \Omega$ is the vacuum impedance, $c$ is the speed of light in vacuum, $\gamma_{0}$ and $\gamma_{f}$ are, respectively, the relativistic Lorentz factor at the beginning and at the end of the linac section, $\bar{\beta}$ is the average betatron function along the linac of length $L_{\text {tot }}$, whose accelerating structures have all length $L_{\mathrm{FODO}} / 2, \Delta$ is the rms offset of the randomly displaced accelerating structures, and $\bar{W}_{\perp}$ is the geometric transverse wake function per unit length computed at $z=2 \sigma_{z}$. The short-range transverse wake function of a cylindrical axis-symmetric structure is given by [23]

$$
W_{\perp}(z)=A\left[1-\left(1+\sqrt{\frac{z}{s_{1}}}\right) \exp \left(-\sqrt{\frac{z}{s_{1}}}\right)\right]
$$

where $A$ and $s_{1}$ depend on the geometry of the accelerating structure's inner cells. For an $S$-band linac, we typically have $s_{1}$ in the range $0.5-0.7 \mathrm{~mm}$ and $A$ in the range $10^{3}-10^{4} \mathrm{~V} / \mathrm{pC} / \mathrm{m}^{2}$

[1] W. A. Barletta, A. M. Sessler, and L.-H. Yu, Nucl. Instrum. Methods Phys. Res., Sect. A 331, 491 (1993).

[2] L.-H. Yu, S. Krinsky, and R. L. Gluckstern, Phys. Rev. Lett. 64, 3011 (1990).

[3] Y.-H. Chin, K.-J. Kim, and M. Xie, LBL Report No. 30673, 1991; Nucl. Instrum. Methods Phys. Res., Sect. A 318, 481 (1992).

[4] M. Xie, in Proceedings of the Particle Accelerator Conference, Dallas, TX, 1995 (IEEE, New York, 1995).

[5] E. L. Saldin, E. A. Schneidmiller, and M. V. Yurkov, in Proceedings of the 26th Free Electron Laser Conference, Trieste, Italy, 2004, edited by R. Bakker, L. Giannessi, M. Marsi and R. Walker (Elettra Sincrotrone Trieste, Trieste, 2004), p. 139, MOPOS15.

[6] R. Bonifacio, C. Pellegrini, and L. Narducci, Opt. Commun. 50, 373 (1984).

[7] A. M. Kondratenko and E. L. Saldin, Part. Accel. 10, 207 (1980).

[8] T. Tanaka, H. Kitamura, and T. Shintake, Nucl. Instrum. Methods Phys. Res., Sect. A 528, 172 (2004).

[9] Y.-V. Chae, A. H. Lumpkin, M. Erdmann, J. W. Lewellen, and S. V. Milton, in Proceedings of the 11th International Free Electron Laser Conference, Trieste, Italy, 2004, edited by R. Bakker, L. Giannessi, M. Marsi and R. Walker (Elettra Sincrotrone Trieste, Trieste, 2004), p. 100, MOBOS03.

[10] S. Di Mitri, Phys. Rev. ST Accel. Beams 16, 050701 (2013).

[11] E. L. Saldin, E. A. Schneidmiller, and M. V. Yurkov, Nucl. Instrum. Methods Phys. Res., Sect. A 528, 355 (2004).

[12] Z. Huang and K.-J. Kim, Phys. Rev. ST Accel. Beams 5, 074401 (2002).

[13] Z. Huang, M. Borland, P. Emma, J. Wu, C. Limborg, G. Stupakov, and J. Welch, Phys. Rev. ST Accel. Beams 7, 074401 (2004).

[14] S. Di Mitri and M. Cornacchia, Phys. Rep. 539, 1 (2014).

[15] A. W. Chao and M. Tigner, Handbook of Accelerator Physics and Engineering, 3rd ed. (World Scientific, Singapore, 2006), p. 66.

[16] S. Reiche et al., Nucl. Instrum. Methods Phys. Res., Sect. A 429, 243 (1999).

[17] M. Venturini and A. Zholents, Nucl. Instrum. Methods Phys. Res., Sect. A 593, 53 (2008).

[18] M. Hüning and H. Schlarb, in Proceedings of the 2003 Particle Accelerator Conference, Portland, OR (IEEE, New York, 2003), WUPAB017.

[19] P. Emma, DESY Report No. TESLA 95-17, 1995.

[20] S. Di Mitri, Ph.D. thesis, Zernike Institute University of Groningen, Netherlands, 2011, ISBN 978-90-367-5176-6.

[21] P. Emma, Report No. LCLS-TN-01-1, 2001. 
[22] K. Flöttmann, T. Limber, and Ph. Piot, DESY Report No. TESLA-FEL-2001-06, 2001.

[23] K. Bane, A. Mosnier, A. Novokhatski, and K. Yokoya, in Proceedings of 1998 International Computational Accelerator Physics Conference, Monterey, CA, 1998, CTH12, http://www.slac.stanford.edu/xorg/icap98/papers/ C-Th12.pdf.

[24] K. L. F. Bane, Report No. SLAC-PUB-11829, 2006.

[25] T. O. Raubenheimer, Phys. Rev. ST Accel. Beams 3, 121002 (2000).

[26] S. Di Mitri and M. Cornacchia, Nucl. Instrum. Methods Phys. Res., Sect. A 735, 60 (2014).

[27] G. Penco, M. B. Danailov, A. Demidovich, E. Allaria, G. De Ninno, S. Di Mitri, W. M. Fawley, E. Ferrari, L. Giannessi, and M. Trovo', Phys. Rev. Lett. 112, 044801 (2014).

[28] E. Allaria et al., Nat. Photonics 7, 913 (2013).

[29] R. Bartolini, Nucl. Instrum. Methods Phys. Res., Sect. A 657, 177 (2011).
[30] G. D'Auria in Proceedings of the Breakdown Science and High Gradient Technology Workshop, Trieste, Italy, 2013 (unpublished), http://indico.cern.ch/conferenceDisplay .py? ovw $=$ True \& confId $=231116$.

[31] W. M. Fawley, Report No. LBNL-49625, 2002.

[32] S. Di Mitri, M. Cornacchia, and S. Spampinati, Phys. Rev. Lett. 110, 014801 (2013).

[33] S. Di Mitri and M. Cornacchia, Nucl. Instrum. Methods Phys. Res., Sect. A 735, 60 (2014).

[34] E. L. Saldin, E. A. Schneidmiller, and M. V. Yurkov, Nucl. Instrum. Methods Phys. Res., Sect. A 398, 373 (1997).

[35] M. Dohlus and T. Limberg, in Proceedings of the 21st Particle Accelerator Conference, Knoxville, TN, 2005 (IEEE, Piscataway, NJ, 2005), TPAT006.

[36] A. W. Chao, Physics of Collective Beam Instabilities in High Energy Accelerators (Wiley, New York 1993), ISBN 0-471-55184-8.

[37] G. Guignard and J. Hagel, Nucl. Instrum. Methods Phys. Res., Sect. A 434, 179 (1999). 